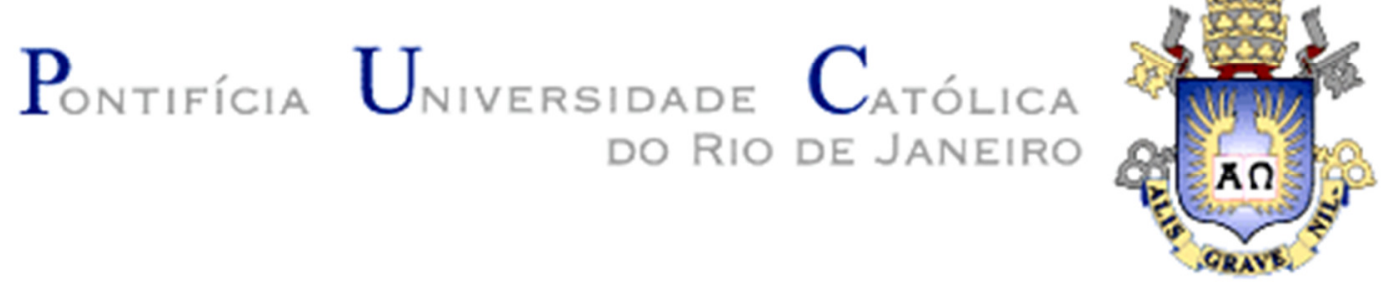

Hyllttonn Wyktor Dhannyells Bazan

Dissertação apresentada como requisito parcial para obtenção do grau de Mestre pelo Programa de PósGraduação em Engenharia Civil do Departamento de Engenharia Civil da PUC-Rio.

Orientador: Prof. Raul Rosas e Silva 


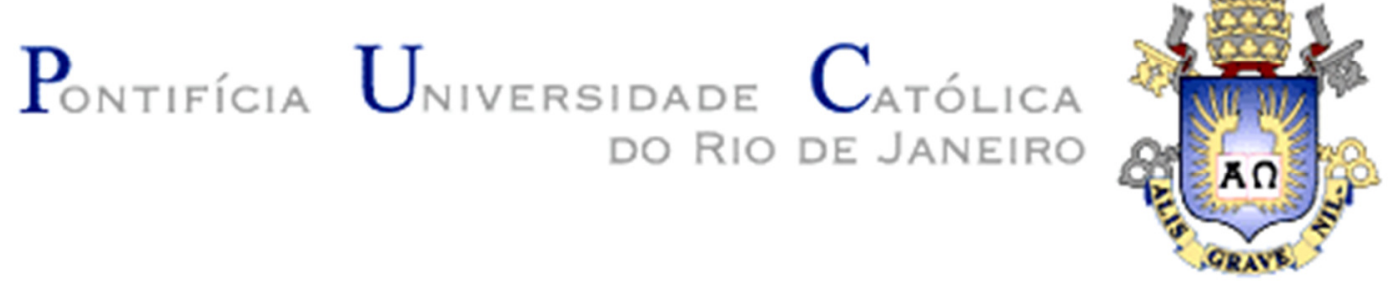

Hyllttonn Wyktor Dhannyells Bazan

\section{Um Modelo de Estaca para Análise de Vibrações}

Dissertação apresentada como requisito parcial para obtenção do grau de Mestre pelo Programa de PósGraduação em Engenharia Civil do Departamento de Engenharia Civil do Centro Técnico Científico da PUCRio. Aprovada pela Comissão Examinadora abaixo assinada.

Prof. Raul Rosas e Silva

Orientador

Departamento de Engenharia Civil - PUC-Rio

Prof. Celso Romanel

Departamento de Engenharia Civil - PUC-Rio

Prof. Paulo Batista Gonçalves

Departamento de Engenharia Civil - PÚC-Rio

Prof. Elvidio Gavassoni Neto

Universidade Federal do Paraná

Prof. José Eugenio Leal

Coordenador Setorial do Centro Técnico Científico - PUC-Rio

Rio de Janeiro, 05 maio de 2015. 
Todos os direitos reservados. É proibida a reprodução total ou parcial do trabalho sem autorização da universidade, do autor e do orientador.

\section{Hyllttonn Wyktor Dhannyells Bazan}

Graduou-se em Engenharia Civil na Universidade Federal do Paraná (UFPR), em Curitiba - Paraná - Brasil, em 2006.

Ficha Catalográfica

Bazan, Hyllttonn Wyktor Dhannyells

Um Modelo de Estaca para Análise de Vibrações / Hyllttonn Wyktor Dhannyells Bazan; orientador: Raul Rosas e Silva. - Rio de Janeiro: PUC, Departamento de Engenharia Civil, 2015.

V., 131 f.: il. (color.); $30 \mathrm{~cm}$

1. Dissertação (mestrado) - Pontifícia Universidade Católica do Rio de Janeiro, Departamento de Engenharia Civil.

Inclui referências bibliográficas.

1. Engenharia Civil - Teses. 2. Sistemas de Iteração Solo-estaca. 3. Frequência Natural. 4. Vibração. 5. Método Rayleigh-Ritz. 6. Testes de Vibração Forçada. 7. Ensaio de Carregamento Dinâmico. 8. Fast Fourier Transform. 9. Domínio da Frequência. I. Silva, Raul R. II. Pontifícia Universidade Católica do Rio de Janeiro. Departamento de Engenharia Civil. III. Título. 
Aos meus pais Daniel e Vicentina que sempre compartilharam meus sonhos e desalentos, vitórias e derrotas, alegrias e tristezas, incentivando-me a prosseguir a jornada, fossem quais fossem os obstáculos, lutando pelo meu sucesso, dedico este trabalho, com a mais profunda admiração e gratidão. 


\section{Agradecimentos}

À Pontifícia Universidade Católica do Rio de Janeiro (PUC-Rio), por ter me nutrido de conhecimento e por ter depositado esperança na minha pessoa. Por ter consentido eu realizar este trabalho em duas campanhas.

Aos professores Raul Rosas e Silva, Deane Roehl e Ney Nascimento pelo conhecimento compartilhado, pela esperança, perseverança e paciência.

Ao meu orientador Professor Raul Rosas e Silva por sua confiança, generosidade, apoio e orientação.

Ao professor Celso Romanel pelo conhecimento compartilhado nas disciplinas de Métodos Numéricos em Engenharia Civil e Dinâmica dos Solos, conhecimentos estes que muito contribuíram para a realização deste trabalho.

Ao Departamento de Engenharia Civil da PUC-Rio, pela oportunidade de ter estudado com professores excepcionais, in memoriam João Luíz Pascal Roehl e Luiz Eloy Vaz, foram professores formidáveis.

À secretária da pós-graduação em Engenharia Civil Rita de Cassia Leite pela paciência e por toda a ajuda que me foi oferecida.

Aos meus pais, pela educação, carinho e incentivo de todas as horas.

Na primeira campanha não posso deixar de agradecer:

Aos amigos Pedro Thá, Marcelo Miqueleto, João Castagnole e Elisangela Oliveira pela acolhida nas primeiras chegadas ao Rio de Janeiro.

À amiga Amanda Jarek por todo o apoio e ajuda nos meses iniciais na 'Casa de Estruturas'. E por ter ajudado na escolha de optar pela pós-graduação da PUCRio.

À família do professor Luiz Eloy Vaz pelo agradável período de convivência que tive oportunidade de ter vivido.

Ao amigo Guilherme Slongo, que provou que a primeira impressão nem sempre é a que fica.

Ao amigo Roberto Machado pela parceria e companhia nos estudos das disciplinas de estruturas e nas longas caminhadas na praia. No inverno carioca algumas figuras se destacam como os pinguins perdidos e os paranaenses.

Aos amigos Elvidio Gavassoni e Jocileia Buback pela amizade, pelas lon conversas e pelos conselhos.

Aos amigos Eduardo Pasquetti, Magnus Meira e José Roberto Silvestre pela ótima convivência em República e fora dela também. 
Aos amigos Jhoran Paredes e Leonardo Erick Cháves pelos ensinamentos em instrumentação geotécnica em rochas e também pelos diversos momentos de descontração.

Às amigas Vivian Marquesi, Lorena Mantovanell e Bernadete Lopes pela agradável e animada convivência na 607-D e fora dela também.

Aos amigos Oscar Fabricio Zuleta Inch e Carlos Aguilera pela valiosa companhia nas disciplinas de estruturas.

Ao Lactec (Instituto de Tecnologia para o Desenvolvimento), na ocasião representado pelas engenheiras Roberta Bomfim Boszczowski e Larissa Ligocki, pelos ensinamentos em instrumentação e monitoramento geotécnico de fundações sob efeitos dinâmicos.

Aos amigos Stenio Barbosa, Kelly Pedrolli, Larissa Passini e André Hebmuller pela amizade e força na primeira campanha e pelo incentivo para a segunda campanha.

Na segunda campanha não posso deixar de agradecer:

Novamente ao amigo Elvidio Gavassoni por me ajudar a iniciar esta segunda campanha.

À querida Nathalia Christina Passos que sem dúvida foi uma das maiores incentivadoras para a finalização desta dissertação, pelo apoio e pela ajuda nos momentos mais difíceis nesse período.

À Fugro In Situ Geotecnia Ltda, representada pelo Diretor Executivo Alessander Morales Kormann e pela Gerente de Testes em Estacas e Fundações Lilian Maier Swinka, pelo incentivo na realização deste trabalho e por todo apoio técnico em teste dinâmicos em estacas fornecido.

Ao amigo Thiago Carnavalle, parceiro e companheiro na disciplina de Dinâmica dos Solos.

Ao amigo José Adriano Malko, parceiro e companheiro na disciplina de Geotecnia Experimental.

Ao CNPQ, à FAPERJ, ao Lactec e à CAPES pelos incentivos concedidos.

Agradeço a Deus pelas pessoas que colocou em minha vida. Todas, sem exceção, contribuíram para a minha formação. 


\section{Resumo}

Bazan, Hyllttonn Wyktor Dhannyells; Rosas e Silva, Raul. Um Modelo de Estaca para Análise de Vibrações. Rio de Janeiro, 2015. 131p. Dissertação de Mestrado - Departamento de Engenharia Civil, Pontifícia Universidade Católica do Rio de Janeiro.

Algumas máquinas produzem solicitações dinâmicas que são transferidas às fundações por meio de movimentos vibratórios. É necessário analisar e estudar esses movimentos vibratórios durante o projeto da fundação para evitar danos aos equipamentos, à própria fundação e às estruturas vizinhas. Este tipo de analise também é muito importante para a segurança e saúde ocupacional de pessoas que venham a frequentar tais estruturas. Por esta razão as frequências naturais de vibração são importantes para o dimensionamento dinâmico de fundações, para se evitar efeitos de ressonância e amplificação de deslocamentos. Neste trabalho, estacas embutidas em fundações elásticas de Winkler foram estudadas. As vibrações longitudinais e transversais foram investigadas. As frequências naturais foram calculadas pelo método de Rayleigh-Ritz considerando estacas como elementos de barra embutidas em fundação elástica no caso da avaliação de vibrações longitudinais, e como vigas embutidas em base elástica no caso da avaliação das vibrações transversais. Na modelagem dos elementos de barra e viga utilizaram-se para aproximações dos deslocamentos funções convencionais enriquecidas com $n$ funções adicionais. As constantes de mola de Winkler foram determinadas por provas de carga verticais e horizontais. Os resultados das vibrações longitudinais foram comparados com medições in situ com estacas reais. Os valores reais da vibração longitudinal das estacas foram obtidos pela aplicação da técnica Fast Fourier Transform nas acelerações resultantes de ensaios de carregamentos dinâmicos, que impõem uma vibração forçada no sistema estaca-solo.

\section{Palavras chave}

Sistemas de Interação Solo-estaca; Frequência Natural; Vibração; Método de Rayleigh-Ritz; Testes de Vibração Forçada; Ensaio de Carregamento Dinâmico; Fast Fourier Transform; Domínio da Frequência. 


\section{Abstract}

Bazan, Hyllttonn Wyktor Dhannyells; Rosas e Silva, Raul (Advisor) A Pile Model for Vibration Analysis. Rio de Janeiro, 2013. 131p. Dissertação de Mestrado - Departamento de Engenharia Civil, Pontifícia Universidade Católica do Rio de Janeiro.

Some machines create dynamic efforts that are transferred to foundations by vibratory movements. Is necessary to analyze and study these movements during foundation design in order avoid damages in equipment, in foundation and in neighbor structures. This analysis is also important to safety and health of persons. For this reason natural vibration frequencies are important to dynamic design of foundations, to avoid resonance effects and displacements amplification. In this work, piles embedded in Winkler elastic foundations were studied. The longitudinal and transversal vibrations were investigated. The natural frequencies are calculated by Rayleigh-Ritz method modelling piles as bars embedded in elastic foundations in the case of longitudinal vibration evaluation, and beams embedded in elastic foundations in the case of transversal vibration evaluation. The bars and beams elements are modeled using conventional shape function enriched with $n$ additional functions. The Winkler spring constants were evaluated by vertical and horizontal load tests. The longitudinal vibration results were compared with in situ measurements in real piles. The longitudinal vibration real values of piles were obtained by Fast Fourier Transform in acceleration data of dynamic load tests that impose a forced vibration in soil-pile system.

\section{Keywords}

Soil-pile Interaction System; Natural Frequency; Vibration; Rayleigh-Ritz Method; Forced Vibration Test; Dynamic Load Testing; Fast Fourier Transform; Frequency Domain. 


\section{Sumário}

1 Introdução 15

1.1. Objetivos 16

1.2. Organização do trabalho 16

2 Vibrações em estacas 18

2.1. Frequência natural de vibração e ressonância 18

2.2. Análise de vibração em estacas 19

2.3. Testes dinâmicos em estacas 20

3 Equações de movimentos 23

3.1. Axiais 23

3.1.1. Frequências de vibrações longitudinais em barras 25

3.2. Transversais $\quad 27$

3.2.1. Vibrações amortecidas 29

3.2.2. Efeito de uma força axial 31

3.2.3. Frequências de vibrações transversais 33

4 Formulação geral 38

4.1. Sistemas com parâmetros discretos e múltiplos graus de liberdade 38

4.2. Sistemas com múltiplos graus de liberdade 42

4.2.1. Forças principais 42

4.2.2. Formulação das equações de movimento 48

4.3. Método de Ritz 56

4.4. Método de Rayleigh $\quad 57$

4.5. Método Rayleigh-Ritz 59

4.6. Formulação hierárquica 63

5 Fundações elásticas $\quad 67$

5.1. Conceito 67

5.2. Determinação do módulo de fundação 68 
6 Análise de respostas dinâmicas no domínio da frequência 71

6.1. Análise espectral 71

6.2. Tipos de sinais $\quad 71$

6.3. Transformada de Fourier (FT) 72

6.4. Transformada de Fourier Discreta 72

6.5. Transformada Rápida de Fourier (FFT) 73

$\begin{array}{ll}7 \text { Resultados } & 75\end{array}$

7.1. Modelagem numérica $\quad 75$

7.1.1. Comparações com soluções analíticas $\quad 75$

7.1.2. Exemplos numéricos $\quad 77$

7.2. Análise espectral 84

7.2.1. Exemplo de FFT em sinal puro de seno 84

ङ 7.2.2. Exemplo de FFT em sinal periódico amortecido 94

7.2.3. Exemplo de FFT aplicado em estacas 95

8 Conclusões e sugestões 102

8.1. Comentários sobre o trabalho 102

$\begin{array}{ll}\text { 8.2. Sugestões } & 103\end{array}$

9 Referências bibliográficas 104

$\begin{array}{lr}\text { Apêndice A } & 108\end{array}$

$\begin{array}{ll}\text { Apêndice B } & 118\end{array}$

$\begin{array}{ll}\text { Apêndice C } & 124\end{array}$ 


\section{Lista de figuras}

Figura 1 - Transdutores de aceleração (direita) e deformação (esquerda) fixados em uma estaca (Cortesia: Fugro In Situ Geotecnia Ltda)

Figura 2 - Estaca instrumentada para ensaio dinâmico (Cortesia: Fugro In Situ Geotecnia Ltda)

Figura 3 - (a) Vibrações axiais em uma barra; (b) forças atuando no elemento 23

Figura 4 - Estaca embutida em solo em um comprimento $L$ 24

Figura 5 - Vibrações transversais de uma viga: (a) elevação da viga; (b) elemento infinitesimal e sua posição em deslocamento; (c) forças atuando em um pequeno elemento

Figura 6 - (a) Vibrações transversais em uma viga, incluindo efeito de amortecimento; (b) forças atuando em um elemento, incluindo amortecimento externo; (c) forças de amortecimento interno e momento resultante

30

Figura 7 - Forças atuando em um pequeno elemento de uma viga submetido a vibrações transversais sob a presença de uma força axial 32 Figura 8 - (a) Pórtico de três níveis; (b) forma do deslocamento; (c) forças atuando ao longo dos graus de liberdade 39

Figura 9 - Seção infinitesimal de uma viga sob efeito de forças $S(x)$ 41 Figura 10 - Forças de inércia em um modelo de massas concentradas de uma viga simplesmente apoiada 43

Figura 11 - Modelo de massa concentradas de uma viga (a) modelo de deslocamentos impostos; (b) forças elásticas requeridas para manter os deslocamentos em (a)

Figura 12 - Modelo de massas concentrada de uma viga (a) modelo de velocidade; (b) forças externas requeridas para balancear forças resistentes causadas pelo amortecimento; (c) modelo de amortecimento

Figura 13 - (a) Vibrações de flexão; (b) forças atuando em um elemento; (c) resistência de amortecimento interno; (d) efeito da força axial 49 
Figura 14 - Vibrações axiais em uma barra: (a) elevação da barra mostrando deslocamentos coordenados; (b) forças atuando no elemento de barra

Figura 15 - Vibrações de flexão de uma viga 58

Figura 16 - Graus de liberdade de um elemento de viga 66

Figura 17 - Viga em base elástica 67

Figura 18 - Módulo de base elástica (a) prova de carga em placa; (b) resultado de um ensaio de prova de carga em placa; (c) aproximação linear do módulo de uma base elástica 69

Figura 19 - Diferença entre sinal analógico e digital 71

Figura 20 - Prova de carga vertical (PI-3) 79

Figura 21 - Prova de carga vertical (PI-10a) 80

Figura 22 - Prova de carga vertical (PI-11) 80

Figura 23 - Prova de carga horizontal (E-105) 82

Figura 24 - Prova de carga vertical (E-105) 83

Figura 25 - FFT (fs=1000 Hz, N=256) 88

Figura 26 - FFT (fs $=5000 \mathrm{~Hz}, \mathrm{~N}=1024)$

Figura 27 - FFT (fs=200 Hz, N=256) 90

Figura 28 - FFT (fs=25 Hz, N=256) 91

Figura 29 - FFT (fs=25 Hz, N=512) 92

Figura 30 - FFT (fs=25.6 Hz, N=512) 93

Figura 31 - Função periódica amortecida e FFT 94

Figura 32 - Acelerações geradas na estaca PI-03 pelos golpes aplicados

96

Figura 33 - Acelerações geradas na estaca PI-10a pelos golpes aplicados

97

Figura 34 - Acelerações geradas na estaca $\mathrm{Pl}-11$ pelos golpes aplicados

Figura 35 - Espectro de acelerações da estaca PI-03 98

Figura 36 - Espectro de acelerações da estaca PI-10a 99

Figura 37 - Espectro de acelerações da estaca PI-11 100 


\section{Lista de tabelas}

Tabela 1 - Faixa de variação do módulo de fundação $\quad 70$

Tabela 2 - Geometria e propriedades físicas de um elemento estrutural 75

Tabela 3 - Vibrações longitudinais $\quad 76$

Tabela 4 - Vibrações transversais $\quad 77$

Tabela 5 - Características geométricas e físicas das estacas 78

Tabela 6 - Provas de carga e coeficientes de mola 79

Tabela 7 - Frequências de vibração longitudinais das estacas $\quad 81$

Tabela 8 - Características geométricas e físicas das estacas 82

Tabela 9 - Provas de carga e parâmetros do solo 83

Tabela 10 - Frequências de vibração transversais e longitudinais $\quad 84$

Tabela 11 - Exemplos de FFT 85

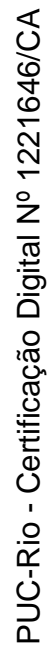

Tabela 12 - Vibração forçada na estaca PI-03 - energias aplicadas $\quad 95$

Tabela 13 - Vibração forçada na estaca PI-10a - energias aplicadas 95

Tabela 14 - Vibração forçada na estaca PI-11 - energias aplicadas 96

Tabela 15 - Frequências naturais longitudinais obtidas pela FFT 101 
Pedi, e vos será concedido; buscai, e encontrareis; batei, e a porta será aberta para vós. Pois todo o que pede recebe; o que busca encontra; e a quem bate, se lhe abrirá. (Mateus 7:8) 